\title{
Comparing Gravitation in Flat Space-Time with General Relativity
}

\author{
Walter Petry \\ Mathematical Institute, University of Düsseldorf, Düsseldorf, Germany \\ Email: wpetry@meduse.de
}

Received 18 July 2016; accepted 21 August 2016; published 24 August 2016

Copyright (C) 2016 by author and Scientific Research Publishing Inc.

This work is licensed under the Creative Commons Attribution International License (CC BY). http://creativecommons.org/licenses/by/4.0/

(c) (i) Open Access

\begin{abstract}
General relativity (GR) and gravitation in flat space-time (GFST) are covariant theories to describe gravitation. The metric of GR is given by the form of proper-time and the metric of GFST is the flat space-time form different from that of proper-time. GR has as source the matter tensor and the Einstein tensor describes the gravitational field whereas the source of GFST is the total energymomentum including gravitation and the field is described by a non-linear differential operator of order two in divergence form. The results of the two theories agree for weak gravitational fields to the order of measurable accuracy. It is well-known that homogeneous, isotropic, cosmological models of GR start from a point singularity of the universe, the so called big bang. The density of matter is infinite. Therefore, our observable universe implies an expansion of space, in particular an inflationary expansion in the beginning. This is the presently most accepted model of the universe although doubts exist because infinities don't exist in physics. GFST starts in the beginning from a homogeneous, isotropic universe with uniformly distributed energy and no matter. In the course of time, matter is created out of energy where the total energy is conserved. There is no singularity. The space is flat and the space may be non-expanding.
\end{abstract}

\section{Keywords}

Gravitation, Cosmology, Flat Space, No Singularity, Non-Expanding Universe

\section{Introduction}

Einstein's general theory of relativity is at present the most accepted theory of gravitation. The theory gives for weak gravitational fields' agreement with the corresponding experimental results. But the results for homogeneous, isotropic, cosmological models imply difficulties. So, the universe starts from a point singularity, i.e. the universe starts from a point with infinite density of matter. The observed universe is very big. Hence, the space 
of the universe must expand very quickly which implies the introduction of an inflationary universe in the beginning.

GFST has a pseudo-Euclidean geometry and the proper time is defined similar to that of general relativity, i.e. space-time and proper time are different from one another. GFST starts from an invariant Lagrangian which gives by standard methods the field equations of gravitation. The source is the total energy-momentum tensor including gravitation. The energy-momentum of gravitation is a tensor. The field is described by non-linear differential equations of order two in divergence form. The theory is generally covariant. The gravitational equations together with the conservation law of the total energy-momentum give the equations of motion for matter. The application of the theory implies for weak gravitational fields the same results as GR to experimental accuracy, e.g. gravitational redshift, deflection of light, perihelion precession, radar time delay, post-Newtonian approximation, gravitational radiation of a two-body system and the precession of the spin axis of a gyroscope in the orbit of a rotation body. But there are also differences of the results of these two theories. GFST gives nonsingular, cosmological models and Birkhoff's theorem doesn't hold. GFST may e.g. be found in the book [1] and in the cited references. Additionally, non-singular, cosmological models are e.g. given in the articles [2]-[6].

Subsequently, homogeneous, isotropic, cosmological models will be summarized. Let us use the pseudoEuclidean geometry. The received universe is non-singular under the assumption that the sum of the density parameters is greater than one, e.g. a little bit greater than one. This implies that the universe may become hot in the course of time. It starts without matter and without radiation and all the energy is gravitational energy. Matter and radiation emerge from this energy by virtue of the conservation of the total energy. The space is flat and the interpretation of a non-expanding space is natural. But it is also possible to state an expansion of space by a suitable transformation as consequence of general covariance of the equations. For a zero cosmological constant matter increases for all times whereas radiation increases and the universe becomes hot. After that radiation decreases to zero as time goes to infinity. Short time after the universe has reached the maximal temperature the production of matter is finished, i.e. the universe appears nearly stationary. Under the assumption of a positive cosmological constant, a certain time after the beginning, matter goes to zero and the universe converges to dark energy as time goes to infinity. Hence, a universe given by GFST appears more natural than that received by GR which gives singular solution with infinite densities. The universe starts from a point and therefore space must expand to be in agreement with the observed big universe. The geometry is in general non-Euclidean but the observed universe implies a flat space.

Section 2 contains GFST; Section 3 contains cosmological models and Section 4 contains the comparison of GFST and GR.

\section{GFST}

The theory of GFST is shortly summarized. The metric is the flat space-time given by

$$
(\mathrm{d} s)^{2}=-\eta_{i j} \mathrm{~d} x^{i}
$$

where $\left(\eta_{i j}\right)$ is a symmetric tensor. Pseudo-Euclidean geometry has the form

$$
\left(\eta_{i j}\right)=(1,1,1,-1) .
$$

Here, $\left(x^{i}\right)=\left(x^{1}, x^{2}, x^{3}\right)$ are the Cartesian coordinates and $x^{4}=c t$. Let

$$
\eta=\operatorname{det}\left(\eta_{i j}\right) \text {. }
$$

The gravitational field is described by a symmetric tensor $\left(g_{i j}\right)$. Let $\left(g^{i j}\right)$ be defined by

$$
g_{i k} g^{k j}=\delta_{i}^{j}
$$

and put similar to (3)

$$
G=\operatorname{det}\left(g_{i j}\right) .
$$

The proper time $\tau$ is defined by

$$
(c \mathrm{~d} \tau)^{2}=-g_{i j} \mathrm{~d} x^{i} \mathrm{~d} x^{j} .
$$


The Lagrangian of the gravitational field is given by

$$
L(G)=-\left(\frac{-G}{-\eta}\right)^{1 / 2} g_{i j} g_{k l} g^{m n}\left(g_{/ m}^{i k} g_{l n}^{j l}-\frac{1}{2} g_{/ m}^{i j} g_{/ n}^{k l}\right)
$$

where the bar/denotes the covariant derivative relative to the flat space-time metric (1).

The Lagrangian of dark energy (given by the cosmological constant $\Lambda$ ) has the form

$$
L(\Lambda)=-8 \Lambda\left(\frac{-G}{-\eta}\right)^{1 / 2} .
$$

Let

$$
\kappa=4 \pi k / c^{4}
$$

where $\kappa$ is the gravitational constant. Then, the mixed energy-momentum tensor of gravitation, of dark energy and of matter of a perfect fluid are

$$
\begin{gathered}
T(G)_{j}^{i}=\frac{1}{8 \kappa}\left[\left(\frac{-G}{-\eta}\right)^{1 / 2} g_{k l} g_{m n} g^{i r}\left(g_{/ j}^{k m} g_{/ r}^{\ln }-\frac{1}{2} g_{l j}^{k l} g_{l r}^{m n}\right)+\frac{1}{2} \delta_{j}^{i} L(G)\right] \\
T(\Lambda)_{j}^{i}=\frac{1}{16 \kappa} \delta_{j}^{i} L(\Lambda) \\
T(M)_{j}^{i}=(\rho+p) g_{j k} u^{k} u^{i}+\delta_{j}^{i} p c^{2} .
\end{gathered}
$$

Here, $\rho, p$ and $u^{i}$ denote density, pressure and four-velocity of matter. It holds by (6)

$$
c^{2}=-g_{i j} u^{i} u^{j} .
$$

Define the covariant differential operator

$$
D_{j}^{i}=\left[\left(\frac{-G}{-\eta}\right)^{1 / 2} g^{k l} g_{j m} g_{/ l}^{m i}\right]_{/ k}
$$

of order two. Then, the field equations for the potentials $\left(g_{i j}\right)$ have the form

$$
D_{j}^{i}-\frac{1}{2} \delta_{j}^{i} D_{k}^{k}=4 \kappa T_{j}^{i}
$$

where

$$
T_{j}^{i}=T(G)_{j}^{i}+T(M)_{j}^{i}+T(\Lambda)_{j}^{i} .
$$

Define the symmetric energy-momentum tensor

$$
T(M)^{i j}=g^{i k} T(M)_{k}^{j}
$$

Then the equations of motion in covariant form are

$$
T(M)_{i / k}^{k}=\frac{1}{2} g_{k l i /} T(M)^{k l} .
$$

In addition to the field Equation (13) and the equations of motion (16) the conservation law of the total energy-momentum holds, i.e.

$$
T_{i / k}^{k}=0 .
$$

The field equations of gravitation are formally similar to those of GR where $T_{j}^{i}$ is the energy-momentum without that of gravitation since the energy-momentum of gravitation is not a tensor for GR. Therefore, the differential operator is the Einstein tensor which may give a non-Euclidean geometry

The results of this chapter may be found in the book [1] and in many other articles of the author, as e.g. in [5]. 


\section{Homogeneous, Isotropic, Cosmological Models}

In this chapter GFST is applied to homogeneous, isotropic, cosmological models. The pseudo-Euclidean geometry (1) with (2) is used. The matter tensor is given by perfect fluid with velocity

$$
u^{i}=0(i=1,2,3)
$$

and pressure $p$ and density $\rho$ with

$$
p=p_{m}+p_{r}, \rho=\rho_{m}+\rho_{r}
$$

where the indices $m$ and $r$ denote matter and radiation. The equations of state for matter (dust) and radiation are

$$
p_{m}=0, p_{r}=\frac{1}{3} \rho_{r}
$$

The potential are by virtue of (18) and the homogeneity and isotropy

$$
g_{i j}=\left\{\begin{array}{lc}
a^{2}(t) & (i=j=1,2,3) \\
-1 / h(t) & (i=j=4) \\
0 \quad & (i \neq j)
\end{array} .\right.
$$

The four-velocity is by Equation (18) and Equation (6)

$$
\left(u^{i}\right)=\left(0,0,0, c h^{1 / 2}\right) .
$$

Let $t_{0}=0$ be the present time and assume as initial conditions at present

$$
a(0)=h(0)=1, \dot{a}(0)=H_{0}, \dot{h}(0)=\dot{h}_{0}, \rho_{m}(0)=\rho_{m 0}, \rho_{r}(0)=\rho_{r 0}
$$

where the dot denotes the time derivative; $H_{0}$ is the Hubble constant and $\dot{h}_{0}$ is a further constant; $\rho_{m 0}$ and $\rho_{r 0}$ denote the present densities of matter and radiation. It follows from (16) under the assumption that matter and radiation do not interact

$$
\rho_{m}=\rho_{m 0} / h^{1 / 2}, \rho_{r}=3 p_{r}=\rho_{r 0} /\left(a h^{1 / 2}\right) .
$$

The field Equation (13) implies by the use of (21) the two nonlinear differential equations

$$
\begin{gathered}
\frac{\mathrm{d}}{\mathrm{d} t}\left(a^{3} h^{1 / 2} \frac{\dot{a}}{a}\right)=2 \kappa c^{4}\left(\frac{1}{2} \rho_{m}+\frac{1}{3} \rho_{r}+\frac{\Lambda}{2 \kappa c^{2}} \frac{a^{3}}{h^{1 / 2}}\right) \\
\frac{\mathrm{d}}{\mathrm{d} t}\left(a^{3} h^{1 / 2} \frac{\dot{h}}{h}\right)=4 \kappa c^{4}\left(\frac{1}{2} \rho_{m}+\rho_{r}+\frac{1}{8 \kappa c^{2}} L(G)-\frac{\Lambda}{2 \kappa c^{2}} \frac{a^{3}}{h^{1 / 2}}\right)
\end{gathered}
$$

where

$$
L(G)=\frac{1}{c^{2}} a^{3} h^{1 / 2}\left(-6\left(\frac{\dot{a}}{a}\right)^{2}+6 \frac{\dot{a}}{a} \frac{\dot{h}}{h}+\frac{1}{2}\left(\frac{\dot{h}}{h}\right)^{2}\right)
$$

The expression $\frac{1}{16 \kappa} L(G)$ is the density of gravitation. The conservation law of the total energy is

$$
\left(\rho_{m}+\rho_{r}\right) c^{2}+\frac{1}{16 \kappa} L(G)+\frac{\Lambda}{2 \kappa} \frac{a^{3}}{h^{1 / 2}}=\lambda c^{2}
$$

where $\lambda$ is a constant of integration. The Equations (25), (26) and (27) give by the use of the initial conditions (23)

$$
\frac{\dot{h}}{h}=-6 \frac{\dot{a}}{a}+2 \frac{4 \kappa c^{4} \lambda t+\varphi_{0}}{2 \kappa c^{4} \lambda t^{2}+\varphi_{0} t+1}
$$

with 


$$
\varphi_{0}=3 H_{0}\left(1+\frac{1}{6} \frac{\dot{h_{0}}}{H_{0}}\right)
$$

Integration of (28) yields

$$
a^{3} h^{\frac{1}{2}}=2 \kappa c^{4} \lambda t^{2}+\varphi_{0} t+1
$$

Equation (27) gives for the present time $t_{0}=0$ by the use of the initial conditions (23)

$$
\frac{1}{3}\left(8 \kappa c^{4} \lambda-\varphi_{0}^{2}\right)=4\left[\frac{8}{3} \pi k\left(\rho_{m 0}+\rho_{r 0}+\frac{\Lambda c^{2}}{8 \pi k}\right)-H_{0}^{2}\right] .
$$

It follows from (27) by the use of the standard definition of the density parameters of matter, radiation and the cosmological constant with the abbreviation

$$
K_{0}=\left(\Omega_{m}+\Omega_{r}+\Omega_{\Lambda}\right) / \Omega_{m}
$$

the differential equation

$$
\left(\frac{\dot{a}}{a}\right)^{2}=\frac{H_{0}^{2}}{\left(2 \kappa c^{4} \lambda t^{2}+\varphi_{0} t+1\right)^{2}}\left[-\Omega_{m} K_{0}+\Omega_{r} a^{2}+\Omega_{m} a^{3}+\Omega_{\Lambda} a^{6}\right] .
$$

The initial condition is by (23)

$$
a(0)=1 .
$$

The solution of (33) with (30) describes a homogeneous, isotropic, cosmological model by GFST.

Relation (31) can be rewritten in the form

$$
\frac{8 \kappa c^{4} \lambda}{H_{0}^{2}}-\left(\frac{\varphi_{0}}{H_{0}}\right)^{2}=12 \Omega_{m} K_{0} .
$$

A necessary and sufficient condition to avoid singular solutions of (33) is

$$
K_{0}>0
$$

which yields

$$
2 \kappa c^{4} \lambda t^{2}+\varphi_{0} t+1>0
$$

for all $t \in \mathbb{R}$. Hence, condition (35) implies a non-singular solution for all $t \in \mathbb{R}$, i.e. we get a non-singular cosmological model. It exists a $t_{1}<t_{0}=0$ such that

$$
\dot{a}\left(t_{1}\right)=0 \text {. }
$$

Put $a_{1}=a\left(t_{1}\right)$ then it follows from (33a) with $t=t_{1}$

$$
\Omega_{r} a_{1}^{2}+\Omega_{m} a_{1}^{3}+\Omega_{m} a_{1}^{6}=\Omega_{m} K_{0} .
$$

It holds for all $t \in \mathbb{R}$

$$
a(t) \geq a_{1}>0 .
$$

Subsequently assume

$$
a_{1} \ll a(0)=1 \text {. }
$$

Then we get by virtue of (38)

$$
K_{0} \ll 1
$$

It follows from (32) by virtue of (41)

$$
\Omega_{r}+\Omega_{m}+\Omega_{\Lambda}=1+\Omega_{m} K_{0},
$$


i.e. the sum of the density parameters is a little bit greater than one. Hence, $a(t)$ starts from a positive value, decreases to a small positive value, and then increases for all $t \in \mathbb{R}$.

The proper time from the beginning of the universe till time $t$ is

$$
\tilde{\tau}(t)=\int_{-\infty}^{t} 1 / h^{1 / 2}(t) \mathrm{d} t .
$$

The differential Equation (33a) is rewritten by the use of (30) in the form

$$
\left(\frac{\dot{a}}{a}\right)^{2}=H_{0}^{2} \frac{1}{h}\left(-\frac{\Omega_{m} K_{0}}{a^{6}}+\frac{\Omega_{r}}{a^{4}}+\frac{\Omega_{m}}{a^{3}}+\Omega_{\Lambda}\right) .
$$

Hence, the differential equation for the function $a$ by the use of the proper time is

$$
\left(\frac{1}{a} \frac{\mathrm{d} a}{\mathrm{~d} \tau}\right)^{2}=H_{0}^{2}\left(-\frac{\Omega_{m} K_{0}}{a^{6}}+\frac{\Omega_{r}}{a^{4}}+\frac{\Omega_{m}}{a^{3}}+\Omega_{\Lambda}\right) .
$$

This differential equation is by virtue of (41) and a not too small function $a(t)$ identical with that of GR for a flat homogeneous, isotropic universe. Therefore, away from the beginning of the universe, the result for the universe agrees for GFST with that of GR.

These results may be found in the book [1] and in the article [5].

The subsequent considerations can be found in the book [1].

We introduce in addition to the proper time $\tilde{\tau}$ the absolute time $t^{\prime}$ by

$$
\mathrm{d} t^{\prime}=\frac{1}{a(t) h^{1 / 2}(t)} \mathrm{d} t=\frac{1}{a(t)} \mathrm{d} \tilde{\tau} .
$$

This gives for the proper time in the universe

$$
(c \mathrm{~d} \tau)^{2}=-a(t)^{2}\left[|\mathrm{~d} x|^{2}-\left(\mathrm{d} c t^{\prime}\right)^{2}\right]
$$

where $|\mathrm{d} x|$ denotes the Euclidean norm of the vector $\mathrm{d} x=\left(\mathrm{d} x_{1}, \mathrm{~d} x_{2}, \mathrm{~d} x_{3}\right)$.

Relation (47) implies that the absolute value of the light-velocity is equal to vacuum light-velocity $c$ for all times $t^{\prime}$.

The introduction of the absolute time $t^{\prime}$ in the differential Equation (45) gives

$$
\left(\frac{\mathrm{d} a}{\mathrm{~d} t^{\prime}}\right)^{2}=\frac{H_{0}^{2}}{a^{2}}\left(-\Omega_{m} K_{0}+\Omega_{r} a^{2}+\Omega_{m} a^{3}+\Omega_{\Lambda} a^{6}\right) .
$$

Assume that a light ray is emitted at distance $r$ at time $t_{e}^{\prime}$ resp. at time $t_{e}^{\prime}+\mathrm{d} t_{e}^{\prime}$ and it is received by the observer at time $t^{\prime}$ resp. at time $t^{\prime}+\mathrm{d} t^{\prime}$. Then, it follows

$$
r=\int_{t_{e}^{\prime}}^{t^{\prime}} c \mathrm{~d} t^{\prime}=c\left(t^{\prime}-t_{e}^{\prime}\right), r=\int_{t_{e}^{\prime}+\mathrm{d} t_{e}^{\prime}}^{t^{\prime}+\mathrm{d} t^{\prime}} c \mathrm{~d} t^{\prime}=c\left(t^{\prime}+\mathrm{d} t^{\prime}-t_{e}^{\prime}-\mathrm{d} t_{e}^{\prime}\right) .
$$

These two equations imply

$$
\mathrm{d} t^{\prime}=\mathrm{d} t_{e}^{\prime}
$$

The age of the universe since the minimal value of $a(t)$ measured with absolute time $t^{\prime}$ till now

$$
\begin{aligned}
\Delta t^{\prime} & =\int_{t_{1}^{\prime}}^{t_{0}^{\prime}} \mathrm{d} t^{\prime}=\int_{a_{1}}^{1} 1 /\left(\frac{\mathrm{d} a}{\mathrm{~d} t^{\prime}}\right) \mathrm{d} a=\frac{1}{H_{0}} \int_{a_{1}}^{1} a \mathrm{~d} a /\left(-\Omega_{m} K_{0}+\Omega_{r} a^{2}+\Omega_{m} a^{3}+\Omega_{\Lambda} a^{6}\right)^{1 / 2} \\
& \geq \frac{1}{H_{0}} \int_{a_{1}}^{1} a \mathrm{~d} a /\left(-\Omega_{m} K_{0}+\left(\Omega_{r}+\Omega_{m}+\Omega_{\Lambda}\right) a^{2}\right)^{1 / 2} \approx \frac{1}{H_{0}} .
\end{aligned}
$$

Therefore, the age of the universe measured with absolute time is greater than $\frac{1}{H_{0}}$ independent of the density parameters, i.e. there is no age problem.

We will now calculate the redshift of light emitted from a distant object at rest and received by the observer at present time. It is useful to introduce the absolute time. Assume that an atom at a distant object emits a photon at 
time $t_{e}^{\prime}$. It follows from relation (46)

$$
\mathrm{d} \tilde{\tau}=a\left(t_{e}^{\prime}\right) \mathrm{d} t^{\prime} .
$$

Therefore, the energy of the emitted photon is

$$
E \sim-g_{44} \frac{\mathrm{d} t^{\prime}}{\mathrm{d} \tau} \sim a\left(t_{e}^{\prime}\right) E_{0} .
$$

The energy of the photon moving to the observer in the universe is constant by virtue of (47), i.e. by the constant light velocity. Then, the corresponding received frequency is

$$
v=a\left(t_{e}^{\prime}\right) v_{0}
$$

where $v_{0}$ is the frequency emitted at the observer from the same atom. The redshift is given by

$$
z=v_{0} / v-1=1 / a\left(t_{e}^{\prime}\right)-1 \text {. }
$$

Light emitted at distance $r$ at time $t_{e}^{\prime}$ and received at $r=0$ at time $t_{0}^{\prime}$ has by the constant velocity of light the relation

$$
r=c\left(t_{0}^{\prime}-t_{e}^{\prime}\right) .
$$

This gives by Taylor expansion of $a\left(t_{e}^{\prime}\right)$ in relation (51)

$$
z=H_{0} \frac{r}{c}+\left(1-\frac{1}{2} \frac{1}{H_{0}^{2}} \frac{\mathrm{d}^{2} a\left(t_{0}^{\prime}\right)}{\mathrm{d} t_{0}^{\prime 2}}\right)\left(H_{0} \frac{r}{c}\right)^{2}
$$

Differentiation of Equation (48) yields by neglecting small expressions

$$
\frac{\mathrm{d}^{2} a\left(t_{e}^{\prime}\right)}{\mathrm{d} t_{e}^{\prime 2}} \approx H_{0}^{2}\left(1-\frac{1}{2} \Omega_{m}+\Omega_{\Lambda}\right)
$$

This gives the redshift formula

$$
z=H_{0} \frac{r}{c}+\frac{3}{4} \Omega_{m}\left(H_{0} \frac{r}{c}\right)^{2} .
$$

The detailed calculations of Formula (52) can be found in the book [1].

Higher order Taylor expansion gives higher order redshift approximations.

\section{Differences of Theory and Results of GFST and GR}

1) It is worth to mention that the space of the universe by GFST is also flat by the use of (6) with (21). This is important because the experiment implying flatness of space of GR uses Formula (6). This is the result of the flat space-time geometry of GFRS. The results for the universe of GFST and GR away from the beginning of the universe agree for a flat space.

2) The metric of GFST is a flat space-time and the space of GFST is flat by the use of (1) and (2). The gravitational field is a tensor of rank two and it is described in flat space-time. The left hand side is a non-linear differential operator of order two and the right hand side is the total energy-momentum tensor including that of gravitation which is a tensor in GFST. Proper time is defined by the use of the gravitational field. The metric of GR is identical with the definition of the proper time which is formally identical with that of GFST. The energy-momentum of gravitation by GR is not a tensor. The left hand side of the field equations is a linear combination of the Ricci tensor and the right hand side of the differential equations is the matter tensor. Both gravitational theories are covariant. The theory of GR implies in general a non-Euclidean geometry. Experimental results indicate that our universe is flat

3) The space of the universe by GFST is by (1) and (2) non-expanding. Experimental results of Lerner [7] also yield a non-expanding universe. The space of the universe by GR is singular in the beginning, i.e. it starts from a point. The observed universe is very big. Therefore, the space must expand and perhaps it implies an inflationary universe. 
4) The universe received by GFST is non-singular, i.e. all the physical quantities are defined in contrast to those of GR where the universe starts with a singularity in the beginning, i.e. the space consists of a point with infinite density of matter.

5) The redshift is an intrinsic gravitational effect by GFST whereas GR explains the redshift as Doppler effect of an expanding universe.

6) Linear perturbation theory of cosmological models by GFST can give in the matter dominated universe a quick increase of the inhomogeneity (see [1], chapter 9.4) which may explain the galaxies whereas by the use of GR the increase of the inhomogeneity is much too slow.

7) The theory of GFST gives non-expanding, cosmological models. Hence, gravitational waves cannot be generated in the beginning. In the beginning of the universe by GR, it can imply gravitational waves by virtue of inflation. Signals from the birth of the universe were measured by BICEP2. But shortly after this announcement the result was retracted.

8) Studies of supernovae are used to measure distances in space. It seems that the ancient supernovae aren't as distant as believed. This means that the cosmological constant is smaller than till now assumed. A vanishing cosmological constant (no dark energy) is perhaps not excluded if a modified Hubble law is used where it is assumed that every object is surrounded by a medium (see [1] chapter 12.4 and article [8]). This gives a new redshift formula.

9) A non-singular, non-expanding universe with vanishing cosmological constant is already studied in article [9].

\section{References}

[1] Petry, W. (2014) A Theory of Gravitation in Flat Space-Time. Science PG.

[2] Petry, W. (1981) General Relativity Gravitation, 13, 1057-1071. http://dx.doi.org/10.1007/BF00756365

[3] Petry, W. (1990) General Relativity Gravitation, 22, 1045-1965. http://dx.doi.org/10.1007/BF00757815

[4] Petry, W. (1997) Astrophysics Space Science, 254, 305-317. http://dx.doi.org/10.1023/A:1000938931517

[5] Petry, W. (2013) Journal Modern Physics, 4, 20-25. http://dx.doi.org/10.4236/jmp.2013.47A1003

[6] Petry, W. (2014) Journal Applied Mathematics Physics, 2, 50-54. http://dx.doi.org/10.4236/jamp.2014.25007

[7] Lerner, E. (2005) arXiv: astro-ph/0509611.

[8] Petry, W. (2013) Physics Essays, 26, 315-320. http://dx.doi.org/10.4006/0836-1398-26.2.315

[9] Petry, W. (2015) Journal Modern Physics, 6, 1085-1094. http://dx.doi.org/10.4236/jmp.2015.68113

Scientific Research Publishing

\section{Submit or recommend next manuscript to SCIRP and we will provide best service for you:}

Accepting pre-submission inquiries through Email, Facebook, LinkedIn, Twitter, etc.

A wide selection of journals (inclusive of 9 subjects, more than 200 journals)

Providing 24-hour high-quality service

User-friendly online submission system

Fair and swift peer-review system

Efficient typesetting and proofreading procedure

Display of the result of downloads and visits, as well as the number of cited articles

Maximum dissemination of your research work

Submit your manuscript at: http://papersubmission.scirp.org/ 